\title{
METASTABLE PHASE FORMATION BY ION BEAM MIXING
}

\author{
G.S. WAS and J.M. ERIDON \\ Department of Nuclear Engineering, University of Michigan, Ann Arbor, MI 48109, USA
}

There are essentially four basic types of metastable alloys which may be formed through heavy ion irradiation of crystalline structures: amorphous phases with no long range order; crystalline phases with structures different from that of the stable intermetallic alloy; disordered crystalline phases with structures based on the same lattice as that of the stable intermetallic; and a quasicrystalline structure. With the exception of the quasicrystalline structure, all of these metastable structures are produced by ion beam mixing of nickel-aluminum alloys with $500 \mathrm{keV}$ krypton ions. Ion beam mixing was performed on samples formed by alternate evaporation of layers of nickel and aluminum as well as on the intermetallic compounds at both 80 and $300 \mathrm{~K}$. The structure resulting from ion beam mixing depended strongly on composition, and hence its formation was governed primarily by thermodynamic considerations. The thermodynamically favored state was determined analytically using the embedded atom method, and the model results are in qualitative agreement with observations of metastable phase formation. However, kinetic considerations are needed to explain the dependence of the final structures on initial structure and temperature.

\section{Introduction}

An effective method of forming nonequilibrium or metastable phases in alloys is ion beam mixing. With this technique, metastable alloys which were previously inaccessible can be readily produced and examined. Since the term "metastable" implies a phase with a free energy higher than that of the stable phase under the prevailing conditions of temperature and pressure, it is natural to look to thermodynamics for an explanation. Experimental results seem to indicate a strong role of thermodynamics in the tendency to form metastable phases. Intermetallic compounds with small ranges of solubility and complex crystal structures are prime candidates for transformation to a metastable phase [1]. Also, the change in the free energy of the solid due to the ion induced defect buildup argues for a thermodynamic explanation [2]. However, not all transformations can be explained on a purely thermodynamic basis.

Hung and Mayer [3] provided a concise summary of the role of kinetics in metastable phase formation. They state that at low temperatures ion mixing is similar to a quench process where the atom configurations are essentially determined during the relaxation period following the collision events. Because kinetics are restricted, the formation of complex crystalline structures is unlikely and ion mixing will usually result in solid solutions, simple cubic structures or amorphous structures. The structure of the metastable system is, however, influenced by the equilibrium nature of the system. Those systems with many intermetallics will tend to form amorphous phases while those with no intermetallic alloys show a tendency to form solid solutions in mixing. At high temperature, atom mobility is significant and equilibrium phases will usually form.

Metastable phases formed by irradiation usually occur by one of four types of transformations: order $\rightarrow$ disorder; crystal structure $\mathrm{A} \rightarrow$ crystal structure $\mathrm{B}$; crystal structure $\mathrm{A} \rightarrow$ amorphous; and crystal structure $A \rightarrow$ quasicrystalline. This paper describes work on the $\mathrm{Ni}-\mathrm{Al}$ system which was designed to show the role of thermodynamics and kinetics in the formation of metastable phases under ion irradiation. The nickelaluminum system is an ideal choice for investigating the formation of metastable phases because three of the four transformations just mentioned occur in this system (fig. 1). Experiments were designed to investigate the effects of temperature, ion dose, initial structure and composition on the formation and transformation of alloy structures. This method differs significantly from much of the previous work in this field in that we chose

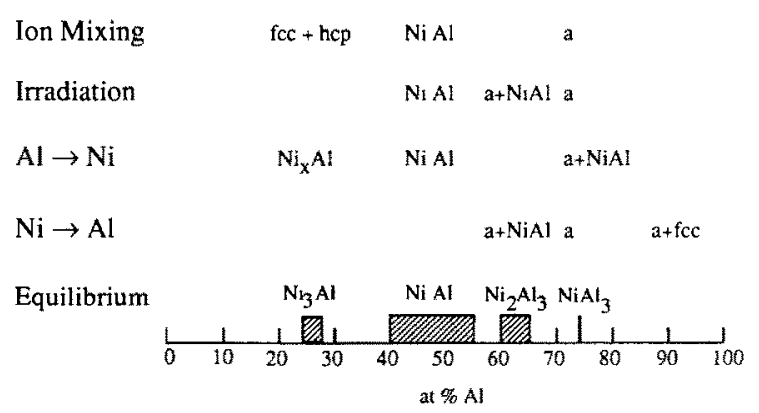

Fig. 1. Summary of $\mathrm{Ni}-\mathrm{Al}$ microstructures vs Al concentration for samples prepared by various ion beam treatments. 
to study only one alloy system as opposed to studying a variety of alloys under similar conditions. By restricting the scope of the investigation to one alloy system at a variety of compositions, it is possible to construct a relatively simple and consistent thermodynamic model of the phases of interest.

\section{Experiment}

Thin film samples of the various compositions were formed by vacuum evaporation of alternate layers of nickel and aluminum onto supported copper grids, forming smooth films with a total thickness of approximately $600 \AA$. The evaporation was performed using a multiple hearth clcctron gun source in a chamber holding a vacuum in the mid $10^{-7}$ Torr range. Half of the samples of each composition were annealed for 1 $\mathrm{h}$ at $723 \mathrm{~K}$ in a vacuum chamber at a pressure of $\sim 10^{-7}$ Torr. This treatment was sufficient to form the stable intcrmctallic compounds found on the phase diagram. The initial grain size of these samples was approximately $100 \AA$, and this did not change appreciably upon annealing.

These samples were irradiated to various doses with $500 \mathrm{keV}$ krypton ions using the $2 \mathrm{MV}$ tandem accelerator in the Materials Science and Technology Division at Argonne National Laboratory. Most of the irradiations were carried out in an ion pumped target chamber holding a vacuum better than $10^{-7}$ Torr. Some irradiations were carried out in the High Voltage Electron Microscope (HVEM) at Argonne. Samples were irradiated at both room temperature $(300 \mathrm{~K})$ and at liquid nitrogen temperature $(80 \mathrm{~K})$. The dose rate for these irradiations was approximately $3 \times 10^{12}$ ions $/\left(\mathrm{cm}^{2} \mathrm{~s}\right)$ which corresponds to an energy deposition rate of about $0.25 \mathrm{~W} / \mathrm{cm}^{2}$. All of the samples irradiated in the HVEM were of the $\mathrm{Ni}-50 \% \mathrm{Al}$ initial composition, some of which were first annealed to form $\beta^{\prime}$. Sample irradiation in the cold stage of the HVEM consisted of Ni-50\% Al layers at both 300 and $130 \mathrm{~K}$, and $\beta^{\prime}(\mathrm{NiAl})$ at 130 K.

Rutherford backscattering spectrometry (RBS) was used in order to check the composition of the samples and also to follow the mixing of the layered samples as a function of ion dose [4]. The spectra indicated that complete mixing was achieved at a dose of $2 \times 10^{16}$ ions $/ \mathrm{cm}^{2}$. Following chamber irradiations, samples were removed from the holder and examined in a JEOL100CX scanning-transmission electron microscope (STEM). The various phases present in each type of sample as a function of ion dose were identified using electron diffraction.

\section{Results}

Samples of composition $\mathrm{Ni}-25 \% \mathrm{Al}$ in the form of an intermetallic and a layered film were ion irradiated at 80 and $300 \mathrm{~K}$. Except in the case of irradiated $\gamma^{\prime}$ at $80 \mathrm{~K}$, the irradiation produced a dual-phase structure consisting of disordered $\gamma^{\prime}$ and a metastable hexagonal ideally close-packed phase with the same interatomic distance as the disordered $\gamma^{\prime}$ phase. Figs. 2A-2C show the results of mixing the layered structure at $300 \mathrm{~K}$ after doses of $5 \times 10^{14}, 2 \times 10^{15}$ and $2 \times 10^{16}$ ions $/ \mathrm{cm}^{2}$, respectively. Several rings which should arise from the hcp structure are not present in the pattern (fig. $2 \mathrm{C}$ ). This is because the phase forms with a preferred orientation such that the [001] direction is parallel to the direction of the ion beam (basal planes parallel to the plane of the foil). The one case in which the hcp phase did not form occurred during irradiation of ordered $\gamma^{\prime}$ at $80 \mathrm{~K}$. In this instance, the stable phase disordered at the lowest dose of $2 \times 10^{14}$ ions $/ \mathrm{cm}^{2}$ and remained disordered up to $2 \times 10^{16}$ ion $/ \mathrm{cm}^{2}$ with no evidence of the formation of either the $\mathrm{NiAl} \beta^{\prime}$ phase or the hcp phase.

At low temperatures ( 80 and $130 \mathrm{~K}$ ), irradiation of samples of $\mathrm{Ni}-50 \% \mathrm{Al}$ composition resulted in the formation of disordered $\mathrm{NiAl} \beta^{\prime}$, regardless of whether the initial sample structure consisted of layers or the ordered intermetallic. Disordering of the intermetallic was incomplete after $1 \times 10^{16}$ ions $/ \mathrm{cm}^{2}$ and the layered structure passed through an amorphous phase after $2 \times 10^{15}$ ions $/ \mathrm{cm}^{2}$ before becoming completely dis-
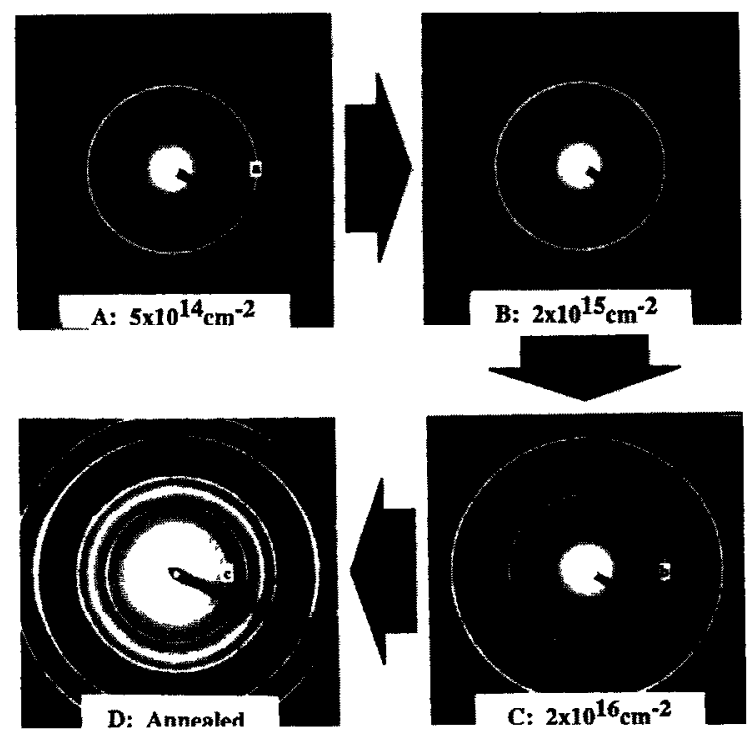

Fig. 2. (A)-(C) Diffraction patterns from layered $\mathrm{Ni}-25 \% \mathrm{Al}$ samples irradiated at $300 \mathrm{~K}$ to various doses; (D) following annealing at $673 \mathrm{~K}$ for $1 \mathrm{~h}$ to form the ordered $\gamma^{\prime}$ alloy. Ring indices: $\mathrm{a}=(111) \gamma ; \mathrm{b}=(100) \mathrm{hcp} ; \mathrm{c}=(100) \gamma$. 

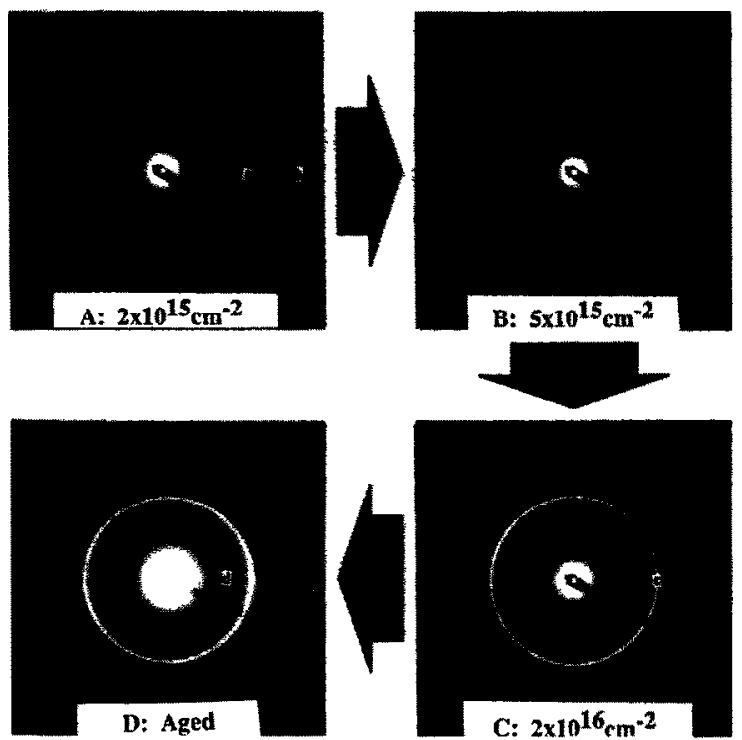

Fig.3 . (A)-(C) Diffraction patterns from layered $\mathrm{Ni}-50 \% \mathrm{Al}$ samples irradiated at $80 \mathrm{~K}$ to various doses; (D) Following aging at room temperature to form the ordered $\beta^{\prime}$ alloy. Ring indices: $a=$ amorphous; $b=(220) \mathrm{Ni} ; \mathrm{c}=(110) \quad \beta ; \mathrm{d}=$ (100) $\beta^{\prime}$.

ordered at $2 \times 10^{16}$ ions $/ \mathrm{cm}^{2}$. Results of mixing the layers at $80 \mathrm{~K}$ are shown in figs. $3 \mathrm{~A}-3 \mathrm{C}$ following doses of $2 \times 10^{15}, 5 \times 10^{15}$ and $2 \times 10^{16}$ ions $/ \mathrm{cm}^{2}$. Ion irradiation of layers at $300 \mathrm{~K}$ causes the layered sample to form the ordered $\beta^{\prime}$ phase.
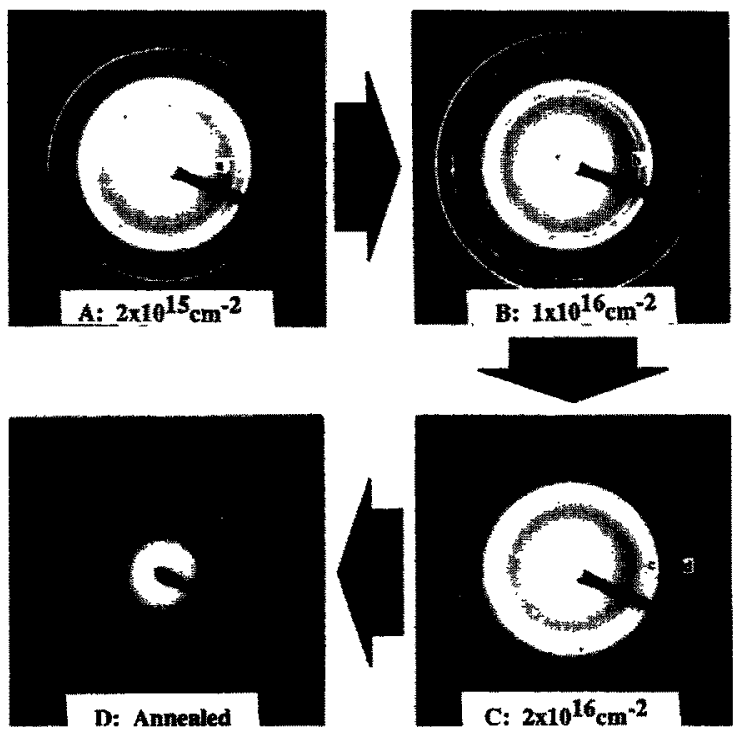

Fig. 4. (A)-(C) Diffraction patterns from layered $\mathrm{Ni}-75 \% \mathrm{Al}$ samples irradiated at $300 \mathrm{~K}$ to various doses; (D) Following annealing at $473 \mathrm{~K}$ for $-10 \mathrm{~s}$ to form the ordered $\epsilon$ alloy. Ring indices: $\mathrm{a}=(100) \beta^{\prime} ; \mathrm{b}=(111) \mathrm{Al} ; \mathrm{c}=$ amorphous; $\mathrm{d}=$ (200) Al.

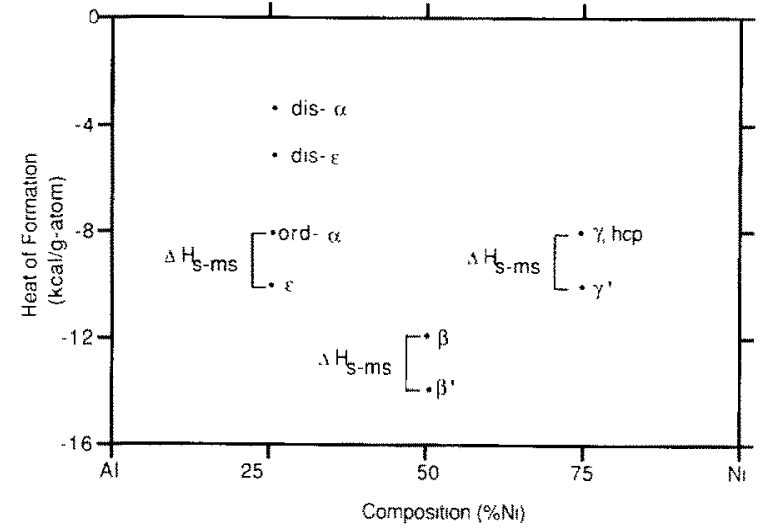

Fig. 5. Heats of formation $\left(\Delta H_{\mathrm{s}}\right)$ and transformation $\left(\Delta H_{\mathrm{s}-\mathrm{ms}}\right)$ for various stable and metastable phases in the $\mathrm{Ni}-\mathrm{Al}$ system. Values for the metastable phases were computed using the embedded atom model.

At $\mathrm{Ni}-75 \% \mathrm{Al}$, all samples formed a two-phase structure containing both elemental aluminum and an amorphous phase, independent of irradiation temperature or initial structure. Annealed samples which were initially of the $\epsilon(\mathrm{D} 0)_{20}$ structure transformed to this two-phase structure at the lowest dose of $2 \times 10^{14}$ ions $/ \mathrm{cm}^{2}$, and remained unchanged as the dose increased to $1 \times 10^{16}$ ions $/ \mathrm{cm}^{2}$. Layers mixed at both 80 and $300 \mathrm{~K}$ also formed this two-phase structure, although a dose of approximately $2 \times 10^{16}$ ions $/ \mathrm{cm}^{2}$ was necessary before the amorphous phase appeared (figs. 4A-4C). The two-phase sample transforms back to the e-phase after only a few seconds at $473 \mathrm{~K}$ (fig. 4D).

\section{Thermodynamic modeling}

In order to analyze the results of the experiments in thermodynamic terms, it is necessary to have some model which can predict the thermodynamic quantities of interest in a metastable phase. For example, it is possible to find measured values of the heats of formation of the various intermetallic alloys in the nickel-aluminum system, but these values are not available for most metastable phases. The model selected for the calculation of thermodynamic quantities of alloys is the embedded atom model. Details of the model are given in ref. [5], and a discussion of its application to the $\mathrm{Ni-Al}$ system is given in refs. [6,7]. The model was first benchmarked against the elastic moduli of the pure elements and then against the heats of formation of the equilibrium phases, $\gamma^{\prime}, \beta^{\prime}$ and $\epsilon$. The model was then used to calculate the heats of formation of the four disordered crystalline phases of interest $\left(\mathrm{Ni}_{3} \mathrm{Al}\left(\gamma^{\prime}\right)\right.$, $\mathrm{Ni}-25 \% \mathrm{Al}$ hcp-phase, $\mathrm{NiAl}\left(\beta^{\prime}\right)$ and $\left.\mathrm{NiAl}_{3}(\epsilon)\right)$, and 
the amorphous phase of composition $\mathrm{NiAl}_{3}$. Results are shown in fig. 5. Two points are important to note. First, the heats of formation of all the metastable phases are greater than those for the corresponding equilibrium phases, in agreement with observation. Second, the heat of formation of the disordered $\gamma^{\prime}$ phase is nearly identical to that of the hcp phase, in agreement with the observation of their coexistence during ion irradiation and their very similar close packed structures. These two results lend support to the validity of the trends predicted by the model.

\section{Discussion}

When the information from fig. 5 is paired with the results of the irradiation experiments, one obvious correlation becomes apparent: metastable states with low heats of transformation $\left(\Delta H_{\mathrm{s}-\mathrm{ms}}\right)$ are easily formed under ion irradiation, while states with high values of $\Delta H_{\mathrm{s}-\mathrm{ms}}$ do not form. In the case of irradiation of samples of ordered $\gamma^{\prime}$, the disordered $\gamma$ phase formed at the lowest dose examined $\left(2 \times 10^{14}\right.$ ions $\left./ \mathrm{cm}^{2}\right)$. The disordered hcp phase, with approximately the same heat of transformation as the disordered $\gamma^{\prime}$ phase, also forms in all cases excepting irradiation of ordered $\gamma^{\prime}$ at $80 \mathrm{~K}$. Irradiation of the ordered $\beta^{\prime}$ phase also produces a disordered structure provided the irradiation occurs at a temperature below about $130 \mathrm{~K}$. Similarly, the amorphous phase formed immediately upon irradiation of the ordered $\epsilon$ phase. In all these cases, the heat of transformation is relatively small, about $1.5 \mathrm{kcal} /(\mathrm{g}$ atoms), and the metastable phase formed at both 80 and $300 \mathrm{~K}$. But, the disordered $\mathrm{NiAl}_{3}$ phase does not form at all since its heat of transformation is over three times as large as that of the amorphous phase at this composition.

While the thermodynamic effect of the size of the heat of transformation $\Delta H_{s-m s}$ is important, it does not explain all of the observed experimental results. In particular, the effects of initial structure on the formation of the hcp phase at $25 \% \mathrm{Al}$ composition, and the effect of temperature on the formation of ordered $\beta^{\prime}$ at equiatomic composition, cannot be explained simply through thermodynamics. Recall that when layers of $25 \% \mathrm{Al}$ composition are mixed at $80 \mathrm{~K}$, the resulting two-phase structures contains both disordered $\gamma^{\prime}$ and a hexagonal phase. However, when the ordered alloy is irradiated at this temperature, the hexagonal phase does not form. The energies of both the disordered $\gamma^{\prime}$ phase and the hexagonal phase are nearly identical, so there is no thermodynamic reason why the hexagonal phase should not form during irradiation of the ordered alloy. Apparently, the initial structure of the sample, layered as opposed to intermetallic compound, has an effect since the hexagonal phase can be formed through irradiation of the ordered intermetallic provided this irradiation takes place at $300 \mathrm{~K}$.

Temperature has a similar effect on the formation of disordered $\beta^{\prime}$ during irradiation of samples of equiatomic composition in that disordered $\beta^{\prime}$ forms at $80 \mathrm{~K}$ but ordered $\beta^{\prime}$ forms at $300 \mathrm{~K}$. In this case, temperature may affect the thermodynamics of phase formation by altering the free energy difference between the ordered and disordered states. Temperature enters into the calculations of free energy explicitly through the entropy term, and since the change in entropy in a disordering reaction is positive, the disordered phase should be favored by higher temperatures. However, the opposite is actually observed. The explanation for these results has to do with the kinetics of the radiation induced transformation.

The effect of radiation is two-fold. First, by knocking atoms off of ordered lattice sites, it produces atomic disorder and an increase in energy of the irradiated material. Second, through both the production of Frenkel defects and the addition of kinetic energy, it enhances diffusion which may lead to ordering and a reduction in energy. The overall effect of the radiation on the state of the material depends on whether diffusive reordering can occur faster than radiation induced disordering. If so, the sample will remain ordered. If not, the energy of the sample will be increased by an amount equal to the heat of transformation from the ordered to the disordered state. This excess energy will be available to drive transformations from the disordered crystalline state to other states. These new states will be metastable, and may be either crystalline, with structures different from that of the original sample, or amorphous.

This model explains in simple terms the observed effect of temperature on the formation of $\beta^{\prime}$ during irradiation. At low temperature $(80 \mathrm{~K})$, the atoms displaced by the radiation cool quickly after coming to rest on lattice sites, reducing the time available for diffusion and leaving the sample in a disordered state. At higher temperaturc $(300 \mathrm{~K})$ the thermalization period is extended to the point where order is restored by diffusion.

The same arguments apply to the formation of the hcp phase at $25 \% \mathrm{Al}$. In order for this phase to form, some diffusion is required to provide the rearrangement necessary for the new crystal structure. This cannot occur at $80 \mathrm{~K}$ where the thermalization period is too brief, but may occur at $300 \mathrm{~K}$. This assumes that the diffusion necessary for the formation of a disordered hcp phase is less than that required for the formation of an ordered fcc phase. While this assumption cannot be easily verified, it is true that the hcp lattice is very similar to the fcc lattice and so the transformation from one lattice to the other should require relatively little atomic rearrangement. The hcp phase may be formed at $80 \mathrm{~K}$ through the mixing of layers of nickel and 
aluminum because the formation of the $\gamma^{\prime}$ phase liberates $\sim 0.33 \mathrm{eV} /$ atom. This energy is released as heat as the layers of nickel and aluminum are mixed by the radiation, and this heat extends the thermalization period of the localized defects, allowing the diffusion necessary for the formation of the hcp phase to occur.

The authors gratefully acknowledge L. Rehn at Argonne National Laboratory for use of the facilities and his many helpful discussions. The work was supported under NSF grants DMR-8310032 and DMR8603174.

\section{References}

[1] J.L. Brimhall, H.E. Kissinger and L.A. Charlot, Radiat. Eff. 77 (1983) 273.

[2] J.L. Brimhall and E.P. Siminen, Nucl. Instr, and Meth. B16 (1986) 187.

[3] L.S. Hung and J.W. Mayer, Nucl. Instr. and Meth. B7/8 (1985) 676.

[4] J.M. Eridon and G.S. Was, Nucl. Instr. and Meth. B12 (1985) 505.

[5] M.S. Daw and M.I. Baskes, Phys. Rev. B29 (1984) 6443.

[6] J.M. Eridon, L. Rehn and G.S. Was, Nucl. Instr. and Meth. B19/20 (1987) 626.

[7] J.M. Eridon, G.S. Was and L. Rehn, submitted to J. Mater. Res. 